\title{
Efecto antinociceptivo del extracto etanólico de las hojas de Maytenus macrocarpa (Ruiz \& Pav.) Briq. "chuchuhuasi” mediante la prueba de contorsiones abdominales en ratones
}

V Robles-Pizarro ${ }^{2}$, L Tarqui-Cabrera, ${ }^{1,2}$ N Rodríguez-Collazos ${ }^{2}$, A Morales-Chau ${ }^{2}$, J De La Cruz-Manyari ${ }^{2}, K_{\text {Kíos- }}$ Melgar 2, D Rivera-Cruzado 2, A Rubio-Gonzáles ${ }^{2}$, C Santa Cruz-Contreras 2, G Velazco-Curay ${ }^{2}$, B Loja-Herrera ${ }^{1}$, A Alvarado-Yarasca ${ }^{1}$, B Castañeda-Castañeda ${ }^{1}$, A Salazar-Granara ${ }^{1,2}$

\section{RESUMEN}

Objetivo: Determinar el efecto y actividad antinociceptiva de las hojas de Maytenus macrocarpa (Ruiz \& Pav) Briq. "chuchuhuasi" mediante la prueba de contorsiones abdominales en roedor.

Material y Métodos: Se utilizaron 40 ratones albinos machos, con pesos medios de $25 \mathrm{~g}$, se empleó la prueba de contorsiones abdominales para determinar la actividad antinociceptiva. El grupo Control, no recibió ninguna sustancia. Se administró extracto etanólico de las hojas de M. macrocarpa (Ruiz \& Pav.) Briq. 2000 mg/kg, Tramadol 10 mg/kg y Diclofenaco sódico $10 \mathrm{mg} / \mathrm{kg}$. Las sustancias fueron administradas por la vía oral una hora antes de la inducción de dolor. Para la validación estadística se usó la prueba de Shapiro-Wilk, ANOVA de una cola, Tukey, y Newman-Keuls.

Resultados: El número de contorsiones abdominales fue 41+/- 3.04, 27+/- 3.55, $9+/$ - 4.14, y 18 +/- 2.65 respectivamente. El porcentaje de inhibición de la conducta nociceptiva fue: $0 \%, 34 \%, 77 \%$, y 55\%. La prueba de ANOVA de una vía, demostró diferencias estadísticas ( $\mathrm{p}<0.05$, IC 95\%), y la prueba de Tukey y Newman-Keuls, demostraron diferencias significativas entre los grupos, frente al control.

Conclusión: Se comprobó el efecto antinociceptivo de las hojas de Maytenus macrocarpa (Ruiz \& Pav.) Briq. “chuchuhuasi”, en dosis de $2000 \mathrm{mg} / \mathrm{kg}$. (Horiz Med 2014; 14(1): 6-10)

Palabras clave: Maytenus macrocarpa, hojas, contorsiones abdominales, dolor, Medicina Tradicional, Plantas Medicinales. (Fuente: DeCS BIREME).

Antinociceptive effect of the ethanol extract of the leaves of Maytenus macrocarpa (Ruiz \& Pav.) Briq. "chuchuhuasi" Through the Writhing Test in mice

\begin{abstract}
Objective: To determine the effect and antinociceptive activity of the leaves of Maytenus macrocarpa (Ruiz \& Pav ) Briq . "Chuchuhuasi " by the writhing test in rodents.

Material and Methods: 40 male albino mice were used, with average weights of $25 \mathrm{~g}$, the writhing test was used to determine the antinociceptive activity. The experimental groups were: Control; received no substance, ethanol extract of the leaves of M. macrocarpa Briq (Ruiz \& Pav.) $2000 \mathrm{mg} / \mathrm{kg}$, Tramadol $10 \mathrm{mg} / \mathrm{kg}$ and $10 \mathrm{mg}$ Sodium Diclofenac $/ \mathrm{kg}$. The substances were administered orally one hour before the induction of pain. For statistical validation the Shapiro -Wilk test, one-tailed ANOVA, Tukey, and Newman -Keuls was used.

Results: Writhing number was $41+/-3.04,27+/-3.55,9+/-4.14$, and $18+/-2.65$ respectively. The inhibition percentage of the nociceptive behavior was: $0 \%, 34 \%, 77 \%$ and $55 \%$. The test of one-way ANOVA showed statistical differences ( $p<0.05$, 95\% Cl), and the Tukey and Newman-Keuls test showed significant differences between groups versus control.

Conclusion: Antinociceptive effect of the leaves of Maytenus macrocarpa (Ruiz \& Pav.) Briq "Chuchuhuasi " was found at doses of $2000 \mathrm{mg} / \mathrm{kg}$. (Horiz Med 2013; 13(4): 6-10)
\end{abstract}

Key words: Maytenus macrocarpa leaves, writhing pain, Traditional Medicine, Medicinal Plants. (Source: MeSH NLM).

Centro de Investigación de Medicina Tradicional y Farmacología, de la Facultad de Medicina Humana de la USMP Lima, Perú.

2 Sociedad Científica de Estudiantes de Medicina de la USMP Lima, Perú. 


\section{INTRODUCCION}

El Perú es uno de los cinco países con la mayor biodiversidad del mundo; ello le representa una condición promisoria para su desarrollo, a través del uso directo de sus recursos naturales, como las plantas medicinales $(1,2)$.

Una planta medicinal, procedente de la Amazonia peruana, es el árbol denominado chuchuhuasi, cuyo nombre científico es Maytenus macrocarpa (Ruiz \& Pav.) Briq., la cual se distribuye en los departamentos de Loreto, Amazonas, Huánuco, Pasco, San Martín, Ucayali y Madre de Dios $(3,4)$.

Tradicionalmente, en el Perú, se reconoce el empleo medicinal de la raíz, la corteza y las hojas de chuchuhuasi, por sus efectos: analgésico, antiinflamatorio, afrodisiaco, antidiarreico, antidisentérico, entre otros $(5,6)$.

Estudios pre-clínicos del género Maytenus, demostraron su efecto antiulceroso, analgésico y antiinflamatorio, de las hojas de las especies brasileiras aquifolium e ilicifolia (7-10).

En el Perú, crece otra especie: Maytenus krukovii, la cual presenta investigaciones de la corteza del árbol, que han demostrado su efecto analgésico y la posible mediación de receptores opioides en esta actividad antinociceptiva $(11,12)$.

Para estas especies, una de las técnicas empleadas para comprobar su efecto analgésico, es la prueba de contorsiones abdominales en roedor $(13,14)$.

Este estudio, explora el posible efecto antinociceptivo en ratones, del extracto etanólico de las hojas de Maytenus macrocarpa (Ruiz \& Pav.) Briq, mediante el método de contorsiones abdominales.

\section{MATERIAL Y MÉTODOS}

Estudio analítico, cuasi-experimental, realizado en el Centro de Investigación de Medicina Tradicional y Farmacología de la Universidad de San Martin de Porres (Lima-Perú).
Se emplearon las hojas de Maytenus macrocarpa (Ruiz \& Pav.) Briq., recolectadas en PucallpaUcayali. La certificación taxonómica y la colecta de la muestra, se realizó bajo los criterios del método de Cerrate, E.1969 (15).

Se emplearon 40 ratones machos Mus musculus, de peso promedio $25 \mathrm{~g}$, procedentes del Centro Nacional de Productos Biológicos del Instituto Nacional de Salud del Perú; estos fueron aclimatados en el bioterio de la Facultad, siguiendo las recomendaciones de procedimientos y bioética del International Guiding Principles for Biomedical Research Involving Animal (16).

Muestra química: Diclofenaco (ampolla de $75 \mathrm{mg} / 3 \mathrm{ml}$ ) lote 090906, Registro Sanitario (RS) $\mathrm{N}^{\circ}$ EG-5956, Tramadol (ampollas de $50 \mathrm{mg} / 1 \mathrm{ml}$ ), lote 080861, RS-N ${ }^{\circ}$ E-17534.

El extracto se preparó siguiendo la metodología descrita por Márquez-Vizcaíno Rita Luz et al (17), así, el material seco y molido de las hojas del Chuchuhuasi (Maytenus macrocarpa), fueron maceradas durante una semana en etanol al $70 \%$ posteriormente la mixtura se filtró y fue puesta en un rotavapor. El producto obtenido se secó en una estufa por un periodo de 48 horas. Este resultante, se molió en el mortero hasta obtener un polvo muy fino, el cual se almacenó en envases herméticos en el refrigerador para su posterior uso. El producto final, fue disuelto en agua destilada, a concentraciones que aseguraron un volumen de administración oral que no superó los $0.25 \mathrm{ml}$ (16).

Prueba de contorsiones abdominales en roedores

Se utilizó el modelo de dolor visceral por ácido acético, prueba nociceptiva. Cada animal recibió una inyección intraperitoneal (I.P.), de $0,1 \mathrm{ml} / 10 \mathrm{~g}$ de peso, de ácido acético al $1 \%(11,12,18)$

Los animales fueron colocados por separado, en jaulas, para luego observar, y contar el número de contorsiones abdominales (distensión exagerada del abdomen y estiramiento de las patas traseras).

Se registró el número total de contorsiones, durante los 20 minutos siguientes a la inyección 
de ácido acético. En la ejecución del experimento, el operador que administró las sustancias, y el operador que realizó el conteo de las contorsiones, desconocían el origen de la misma.

Los grupos experimentales recibieron las sustancias de estudio por vía oral una hora antes de la inducción de dolor.

Grupo 1: Control, no recibió ninguna sustancia.

Grupo 2: Extracto etanólico de las hoj as de Maytenus macrocarpa (Ruiz \& Pav.) Briq. (chuchuhuasi) 2000 $\mathrm{mg} / \mathrm{kg}$.

Grupo 3: Tramadol $10 \mathrm{mg} / \mathrm{kg}$.

Grupo 4: Diclofenaco sódico $10 \mathrm{mg} / \mathrm{kg}$.

Se aplicó el test de normalidad de Shapiro-Wilk, el test de ANOVA de 1 vía y el test de Tukey y de Newman-Keuls, para las comparaciones entre los grupos; se estableció la significancia estadística, para un valor $\mathrm{p}<0.05$, y un intervalo de confianza al $95 \%$; se empleó como soporte informático Microsoft Office Excel 2010, y el programa estadístico GraphPadPrism Versión 5.01.

\section{RESULTADOS}

El grupo 1 (control), presentó una media de contorsiones abdominales de $41.33+/$ - 3.04, frente al grupo 2 (chuchuhuasi) que fue $27.11+/-3.55$; el grupo 3 (tramadol), que fue de $9.22+/-4.14$, y el grupo 4 (diclofenaco sódico), la media fue de 18.56 $+/$ - 2.65. (Ver fig.1.)

La prueba de Shapiro-Wilk para las contorsiones abdominales, tuvo los valores probabilísticos: 0.15 , $0.82,0.73$, y 0.37 . La prueba de ANOVA de una vía, demostró un valor probabilístico de 0.0001 . Las pruebas de apareamiento de Tukey y NewmanKeuls, revelaron un valor probabilístico menor a 0.05 .

El porcentaje de efecto antinociceptivo del dolor, de mayor a menor fue: en primer lugar para el grupo que recibió tramadol $(77.69+/-10.03)$, seguido del grupo que recibió diclofenaco sódico $(55.10$ $+/-6.41)$, y del que recibió chuchuhuasi $(34.40+/$ 8.59).

Mediante la prueba de Shapiro-Wilk, se determinó que la distribución para el porcentaje de inhibición del dolor fue normal, presentando los grupos experimentales que recibieron chuchuhuasi, tramadol y diclofenaco sódico, un valor probabilístico $p>0.05$. La prueba de ANOVA de una cola, determinó un valor $p \varangle 0.0001$. Las pruebas de apareamiento de Tukey y Newman-Keuls presentaron un valor probabilístico menor a 0.05.

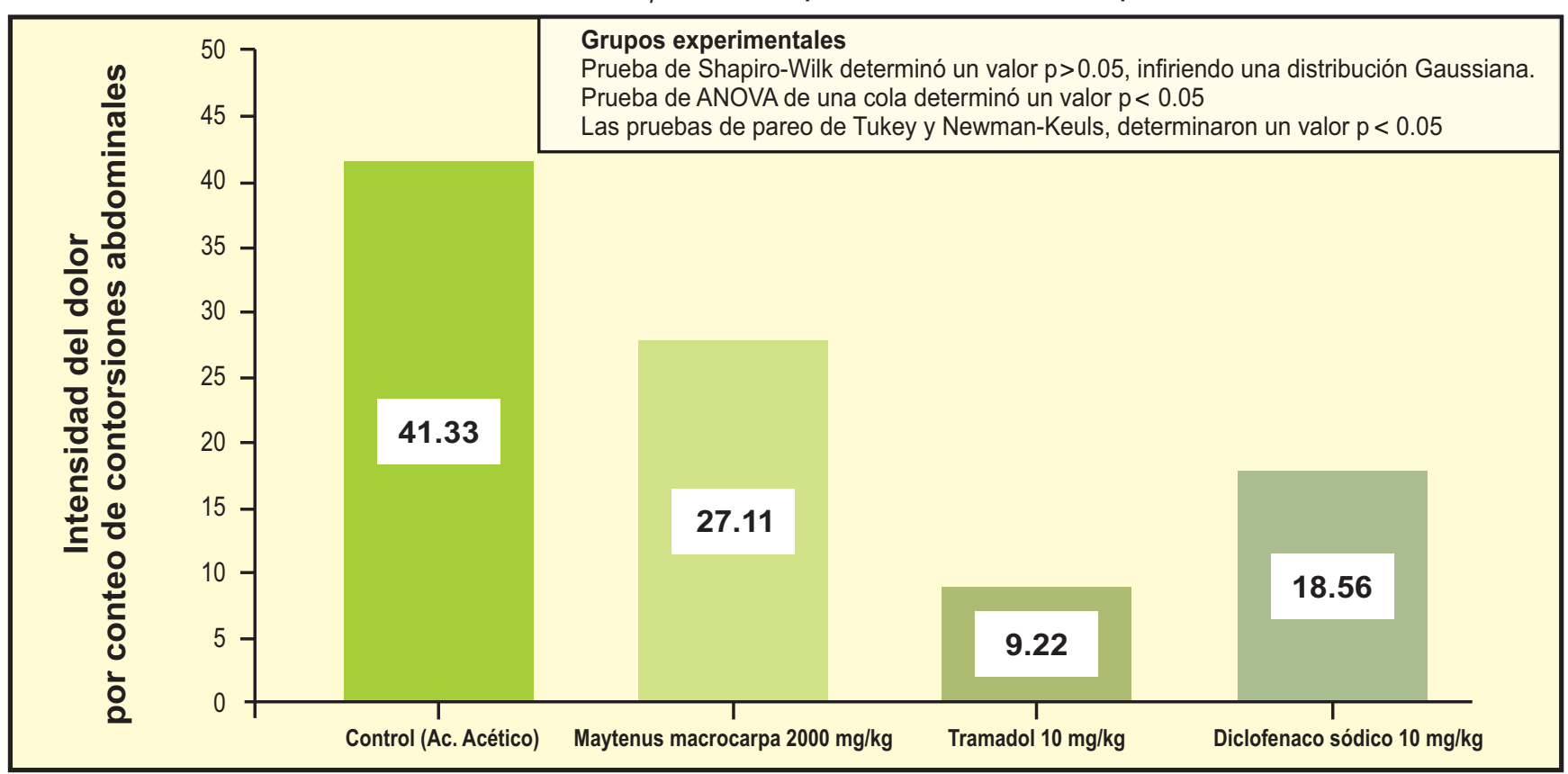

Figura 1. Grupos experimentales 


\section{DISCUSIÓN}

El efecto analgésico de las especies sudamericanas de Maytenus krukovii (corteza), aquifolium (hojas), ilicifolia (hojas), truncata (hoj as), y rígida (corteza), han sido demostrados en trabajos previos (7-12,1922). Este estudio, extendió, y demostró el efecto antinociceptivo, del extracto etanólico de las hojas de Maytenus macrocarpa.

La prueba de contorsiones abdominales, es un modelo experimental de dolor, que implica estímulos algogénicos periféricos, y que por tanto, infieren actividad antinociceptiva en estos niveles, sin ser completamente especifico ni excluir efectos analgésicos en el sistema nervioso central.

La lesión de las membranas celulares peritoneales (que se produce en el test de contorsiones abdominales), genera liberación de prostaglandinas y de otros mediadores de la inflamación, que sustentan inespecíficamente, el potencial efecto antiinflamatorio de una sustancia (14).

De esta manera, en base al efecto antinociceptivo revelado en este estudio, de las hojas de Maytenus macrocarpa, es posible especular que también presente actividad antiinflamatoria, lo cual deberá dilucidarse en nuevos estudios.

Por esto, las investigaciones futuras, deberían buscar la evaluación de las hojas de Maytenus macrocarpa, en otros modelos experimentales de dolor, e inflamación, a fin de delimitar el nivel de acción de la misma y también el mecanismo de acción.

Las hojas de Maytenus truncata, fueron evaluadas en el modelo animal de nocicepción denominado prueba de formalina, demostrando su efecto analgésico en la fase 1 de este modelo, infiriendo participación de vías del dolor neurogénico en la actividad antinociceptiva (21).

En otro estudio, Ias hoj as de Maytenus ilicifolia por la prueba de formaldehido, demostró mejor actividad en la fase 2 del experimento, infiriéndole al extracto de la planta: actividad antiinflamatoria (9).

La corteza de Maytenus rígida, demostró actividad antinociceptiva en el modelo experimental de la retirada de la cola, que también extrapola actividad antinociceptiva en vías del dolor neurogénicas (20).

Considerando la actividad antinociceptiva de las hoj as de Maytenus macrocarpa y de las otras especies revisadas, sería conveniente llevar este conocimiento a la praxis clínica, con un enfoque de prevención de potenciales interacciones con medicamentos, a fin de conocer los efectos indeseados.

\section{Fuentes de financiamiento}

Co-financiamiento entre los autores y la Facultad de Medicina Humana de la USMP.

\section{Conflictos de interés}

Los autores declaran no tener ningún conflicto de interés.

\section{REFERENCIAS BIBLIOGRÁFICAS}

1. Palacios L. Economía y plantas medicinales. Facultad De Ciencias Económicas, Universidad Nacional Mayor de San Marcos. Boletín CSI, 2004; 52(2):163-170.

2. Brak E A. Tratado de Libre Comercio y Biodiversidad en el Perú. [serie en Internet] Perú. [citada Jun 2004, consultada 25 de mayo 2013]. Disponible en: http://bibliotecavirtual. educared.org/index. php/site/ default/detalle/ id/00000000486/tratado-de-libre-comercio-ybiodiversidad-del-Peru .

3. Vega M, Etnobotánica de la Amazonía Peruana.1ra ed. Quito-Ecuador: Ed.Abya-Yala; 2001, 74-76.

4. Schultes R-E, Robert E-R. La selva sanadora: plantas medicinales y tóxicas del noroeste del Amazonas. Revista de Estudios Sociales 2009; 32, 126-142.

5. Villar M, Villavicencio O. Lima, EsSalud; OPS. Manual de Fitoterapia. Capitulo 7: Monografías de plantas medicinales. EsSalud / OPS, 2001;pg 150-153. Disponible en: http://www.bvsde.paho. org/texcom/manualesMEC/fitoterapia/cap7.pdf

6. Mejía K, Rengifo E. Plantas medicinales de uso popular en la amazonia peruana. Agencia Española De Cooperación Internacional (AECI). Primera edición: 1995, pg 71-72. Disponible en: http:// www. iiap. org. pe/ Upload/ Publicacion/ L017. pdf 
7. Ralph Santos-Oliveiral, Simone Coulaud-Cunhall, Waldeciro Colaço. Review of Maytenus ilicifolia Mart. ex Reissek, Celastraceae. Contribution to the studies of pharmacological properties. $\mathrm{Br} \mathrm{J}$ of Pharmacognosy 2009; 19(2B): 650-659,

8. Queiroga C, Faria G, Correa Dias P. Evaluation of the antiulcerogenic activity of friedelan$3 \mathrm{~b}-\mathrm{ol}$ and friedelin isolated from Maytenus ilicifolia(Celastraceae). J of Ethnopharmacol 2000; 11 (3) 465-468.

9. Leite J, Oliveira A, Tagliati C.A. Evaluation of antinociceptive, anti-inflammatory and anti ulcerogenic activities of Maytenus ilicifolia. $\mathrm{J}$ of Ethnopharmacol 2004; 14 (1): 93-100.

10. Gonzalez FG, Portela TY, Stipp EJ, Di Stasi LC. Antiulcerogenic and analgesic effects of Maytenus aquifolium, Sorocea bomplandii and Zolernia ilicifolia. J Ethnopharmacol 2001; 77(1):41-7.

11. Rivas E, Lengua L, Liu H, Salazar A, Román L, Salvador I, Rabanal P, Castañeda B, Manrique R, Ibañez L. Estudio de la actividad analgésica de extractos metanólicos de Maytenus krukovii (Chuchuhuasi), Alchornea castaneifolia (Hiporuro), Sambucus nigra (Saúco), y Aristeguietia discolor (Pulmonaria) en ratones frente al ibuprofeno. Horiz Med 2005; 5(1): 57-61.

12. Salazar A, Santa María J, Zimic C, Salinas I, Sánchez L, Arrambide J, Zapater L, Arias M, Ulffe G, Páucar R, Ibáñez L, Castañeda B. Anti nocicepción opioide del extracto metanólico del Chuchuhuasi (Maytenus krukovii) en un modelo de dolor visceral en ratón. Horiz Med 2006; 6(2): 135-140.

13. International Association for the Study of Pain. Do Animal Models Tell Us about Human Pain?. PAIN CLINICAL UPDATES 2010; XVIII, Issue 5 July

14. Daniel Le Bars, Manuela Gozariu, Samuel W. Cadden. Animal Models of Nociception. Pharmacol Rev 2001; 53:597-652.

15. Cerrate E. Manera de preparar plantas para un herbario. Museo de Historia Natural (Lima). Serie de Divulgación 1969. No.1. 10.

16. International Guiding Principles for Biomedical Research Involving Animals. Androl 1986, 18: 553-554.
17. Márquez-Vizcaíno R, De la Rosa C, Mercado-Pérez A. Actividad antifúngica del extracto total en etanol de las hoj as frescas de Pedilanthus tithymaloides $L$. Poit. (Ultimorrial). Scientia Et Technica 2007; XIII(33):155-159.

18. Siegnund E, Cadmus R. A method for evaluating both non-narcotic and narcotic analgesics. Prog Soc Exp Biol Med 1957; (95):729-731.

19. Oliveira MG, Monteiro MG, Macaúbas C, Barbosa VP, Carlini EA. Pharmacologic and toxicologic effects of two Maytenus species in laboratory animals. J of Ethnopharmacol 1991; 34: 29-41

20. Dias KS, Marques MS, Menezes IA, Santos TC, Silva $A B$, Estevam CS, Sant'Ana AE, Pizza C, et al. Antinociceptive activity of Maytenus rigida stem bark. Fitoterapia 2007; 78, 460-464.

21. Nascentes de Deus A, De Fátima $G$, De Jesus J, Meléndez G, Pains L, Pamplona R, Martinez R, Tagliati C, Leomar C, et al. Estudo fitoquímico do decocto das folhas de Maytenus truncata Reissek e avaliação das atividades antinociceptiva, antiedematogênica e antiulcerogênica de extratos do decocto. Quim Nova 2007; 30 (4): 842-847.

22. Martins M, Estevam C, Santos A, Dias A, Cupertinoda-Silva Y, Araújo-Júnior J, Miranda A, Barreiro E, Pizza C, Piacente S, Montoro P, QuintansJúnior L, Araujo B, Alexandre-Moreira M, Sant'Ana A. Antinociceptive effects of an extract, fraction and an isolated compound of the stem bark of Maytenus rigida. $\mathrm{Br} \mathrm{J}$ of Pharmacognosy 2012; 22(3): 598-603.

\section{Correspondencia:}

Alberto Salazar Granara

Centro de Investigación de Medicina Tradicional y Farmacología, de la FMH-USMP

Dirección: Av. El Corregidor № 1531, Urbanización

Los Sirius, Las Viñas, La Molina, Lima-Perú.

Teléfono: 5113652300 extensión 151/114. Correo electrónico: alberto.salazar@gmail.com

Recibido: 06 de Setiembre de 2013 Aprobado: 06 de Enero de 2014 